\title{
Removal of Unusual Foreign Metal Object (Nail) from the Right Ventricle of Human Heart: A Case Report
}

\author{
Mingliang Zuo, MMed, Qiuyi Chen, BA, Bo Xiang, MMed, Tao Yu, MMed, Lixue Yin, MMed \\ Sichuan Academy of Medical Science \& Sichuan Provincial People's Hospital, Sichuan, China
}

\section{ABSTRACT}

Migration of foreign bodies (FB) with the blood flow to the heart is a rare, but very alarming condition as it may lead to life-threatening complications and death. Objects that are larger than $5 \mathrm{~mm}$ in diameter and/or irregular in shape are recommended for removal from extra- and intracardiac areas to prevent incurable embolization. Surgical extraction of intracardiac objects is a serious surgical challenge associated with difficulties to operate, during the continuous movement of the heart, and identify the exact FB location. Early diagnosis and timely removal of FBs are crucial treatment factors for this rare case resolution. We report a case of accidental migration of a metal FB object (nail) about $1.0^{*} 0.3 \mathrm{~cm}$ from the right neck area jugular vein to the right ventricle apex in the heart. The FB localization was accurately detected using Bi-plane transesophageal echocardiography (TEE) with a special comet-tail artifact. TEE provided valuable information before surgery, and the nail was successfully removed through open-heart surgical procedures and cardiopulmonary bypass (CPB). Postoperative tests indicated no complications.

\section{INTRODUCTION}

Accidental penetration of metallic FB in the heart is rare and has the potential to cause serious complications, including infective endocarditis, thrombosis, pulmonary embolism, and death [Cianciulli 2017; Di Paolo 2015; Liang 2014]. The exact location of the FB not only determines the complications encountered but also helps to ensure complete removal. Therefore, early diagnosis and prompt removal are potentially lifesaving and necessary, even in asymptomatic patients. Here, we describe a case of a cardiac embolization from the jugular vein to the right ventricle, due to accidental injection of a metallic object (nail) without an exit wound. Previously, some researchers found that bullets and needles rapidly migrated from gunshot or injury wounds in the chest

Received December 22, 2020; received in revised form February 21, 2021; accepted February 22, 2021.

Correspondence: Mingliang Zuo, Sichuan Academy of Medical Science \& Sichuan Provincial People's Hospital, Sichuan, China (e-mail: zuoml@ hotmail.com). tissue to the right ventricle. Due to the risk of embolism, past studies have shown that different auxiliary imaging techniques can assist surgical foreign body removal. For instance, the identified FBs can successfully be removed after accurate location using transthoracic echocardiography (TTE), TEE, preoperative $\mathrm{CT}$, and intra-operative TEE, according to past research. In this case, Bi-plane TEE was used to identify the $\mathrm{FB}$ in the apex of the right ventricle (RV) near the diaphragm and eventually helped to successfully remove it. After being found in the human body, how the foreign bodies should be managed depends on the actual individual situation as foreign bodies enter through different paths, which will have different trajectories and eventually lead to different clinical outcomes.

\section{CASE REPORT}

A 30-year-old male, previously healthy, decorative construction worker, was referred to our cardiac center from another medical center, where removal of a metallic foreign body (FB) had failed. When he was using a nail gun to decorate at home, the nail broke, rebounded from the wall, and shot into the right side of his neck. Doctors used a magnet to attract the neck skin and found a foreign body inside, and he was treated at the local hospital. Highdensity shadow was detected in his neck, about $1.1 \mathrm{~cm}$ in length (Figure 1.1). The foreign body in the right neck was removed under local anesthesia, but no foreign body was found. Later, it was located at the bottom of his heart using $\mathrm{X}$-ray (Figure 1.2). The condition of this case was in line with the indications for surgery, so it was decided to perform surgery to remove the foreign body through the cardiopulmonary bypass to prevent further physical damage. The patient had complained of right neck pain since the accident. A wound was found on the right lateral part of his neck, just over the external jugular vein (Figure 2). His chest X-ray from the referral hospital revealed an obliquely oriented nail-shaped foreign body that migrated from the neck to the chest region two hours later after unsuccessful attempts to remove the object. The metallic foreign body was further visualized in the right ventricular using cardiac computed tomography (CT) scan via transverse, sagittal, and coronal positions (Figures 3.1, 3.2, and 3.3). Intra-operative Biplane-TEE (X7-2t probe) showed a $1.0^{*} 0.3 \mathrm{~cm}$ nail without mobility lying in the apical right ventricular near the diaphragm in modified $\mathrm{RV}$ inflow view (Figure 4). The patient remained hemodynamically stable. 


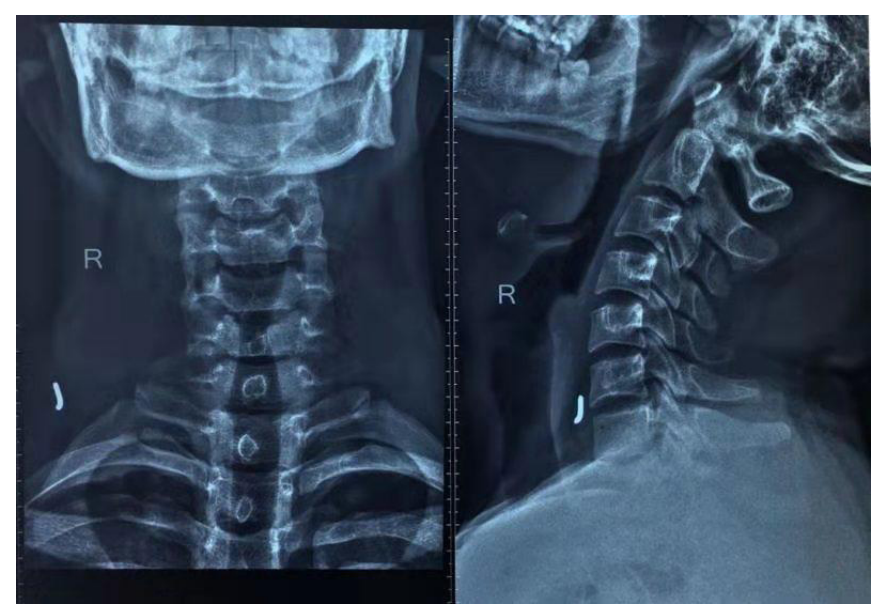

Figure 1.1. A) Metallic foreign body under his neck skin; B) High density shadow at the right side.

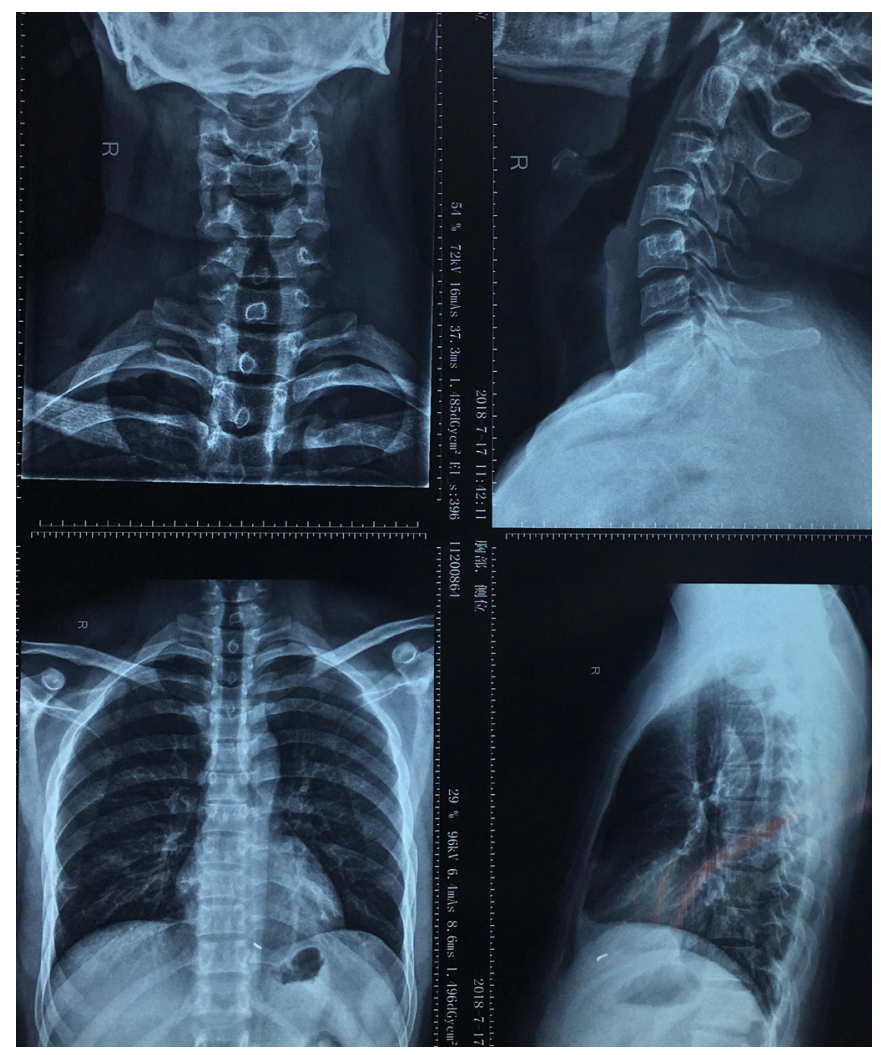

Figure 1.2. The metallic foreign body migrated from his neck to the right ventricle; B) High density shadow at the bottom of the heart.

A median sternotomy was performed, and cardiopulmonary bypass $(\mathrm{CPB})$ was initiated. During surgery, a $1.0 * 0.3$ $\mathrm{cm}$ nail with some clot (Figure 5) was located at the apex of RV and successfully removed from the right atrium. Postoperative transthoracic echocardiography (TTE) showed no abnormality, and the patient was discharged from the hospital one week after surgery without complications.

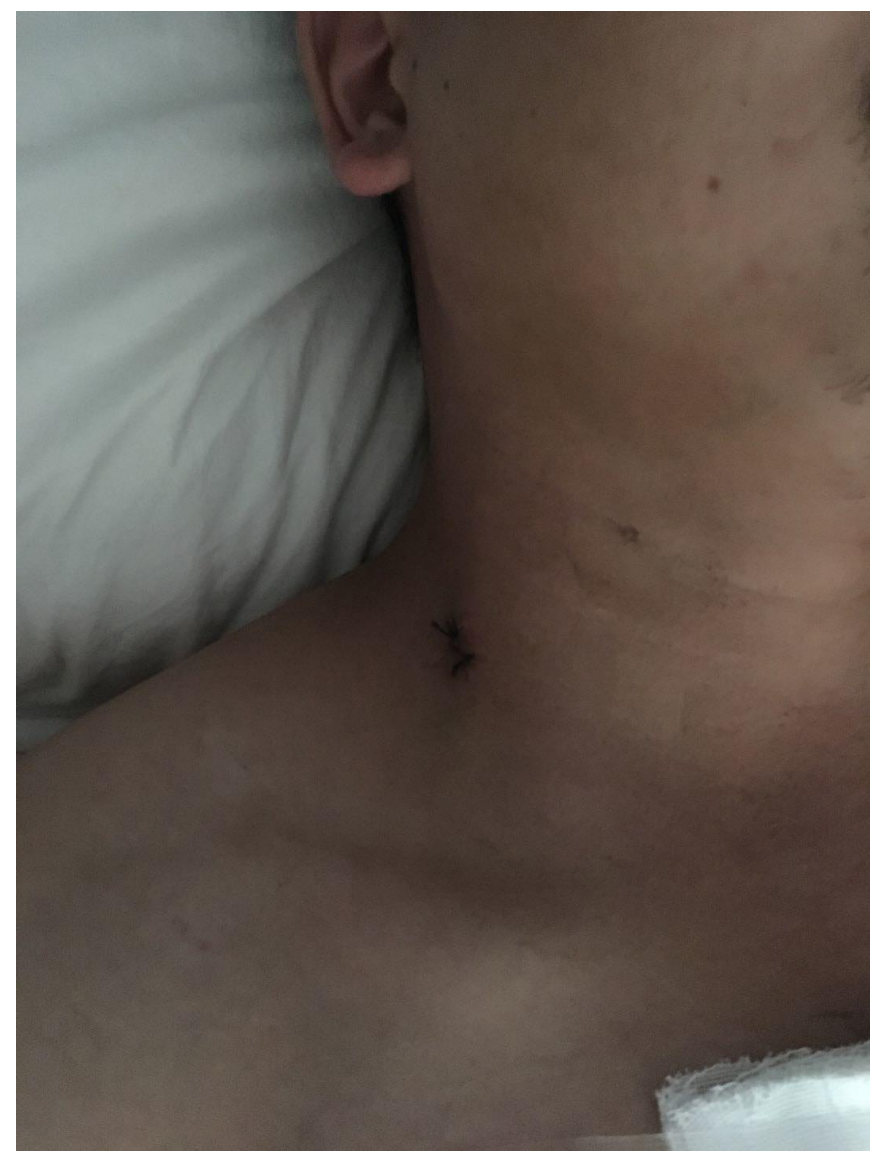

Figure 2. Wound on his right lateral neck.

\section{DIscussion}

Heart foreign body can be located in the pericardial cavity, myocardium, or cardiac cavity. Most foreign bodies in the heart are caused by penetrating trauma to the heart. However, foreign bodies also can enter the heart through the blood flow of the large veins and liver, or the mediastinum via a breach in the esophagus or trachea and then be wrapped and fixed by the fibrous tissue, which can cause no symptoms for life. In this case, the broken nail entered the right heart through the peripheral vein and finally was located at the bottom of the right ventricle. However, a free foreign body in the heart cavity may escape with the blood, having risk of lower lobe pulmonary embolism, bacterial endocarditis or posttraumatic pericarditis, which makes the immediate implementation of surgery necessary. Therefore, surgical removal of the foreign body is beneficial to control infection and avoid further damage to the heart.

Previous case reports described surgical removal of penetrating $\mathrm{FB}$ in the heart, including bullets, acupuncture needles, venous catheter, and the sewing needle [Actis 2003; Di Paolo 2015; Irdem 2015; Supomo 2018]. Reviewing the relevant literature, we found no reports about cardiac embolism caused by a metallic structural nail that migrated from a peripheral vein and entered the patient's heart after an accidental neck injury caused by the use of a high-speed nail gun. 


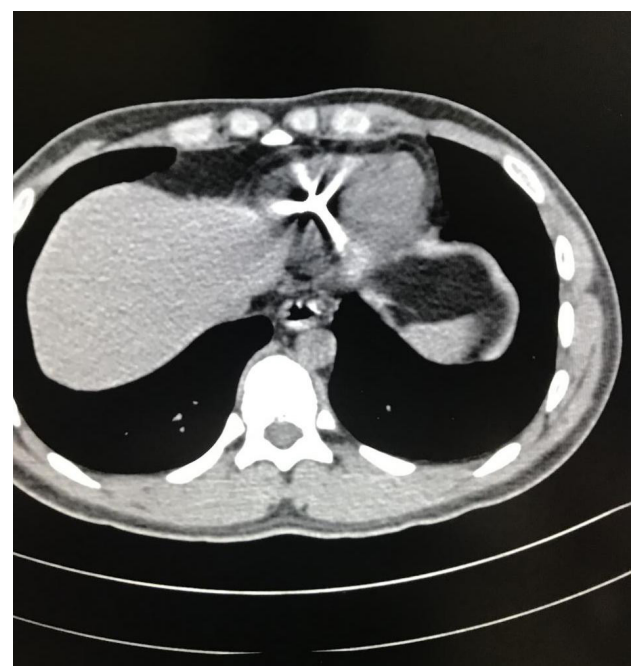

Figure 3.1. (axial scan) Cardiac computed tomography showed the FB in right ventricular chamber.

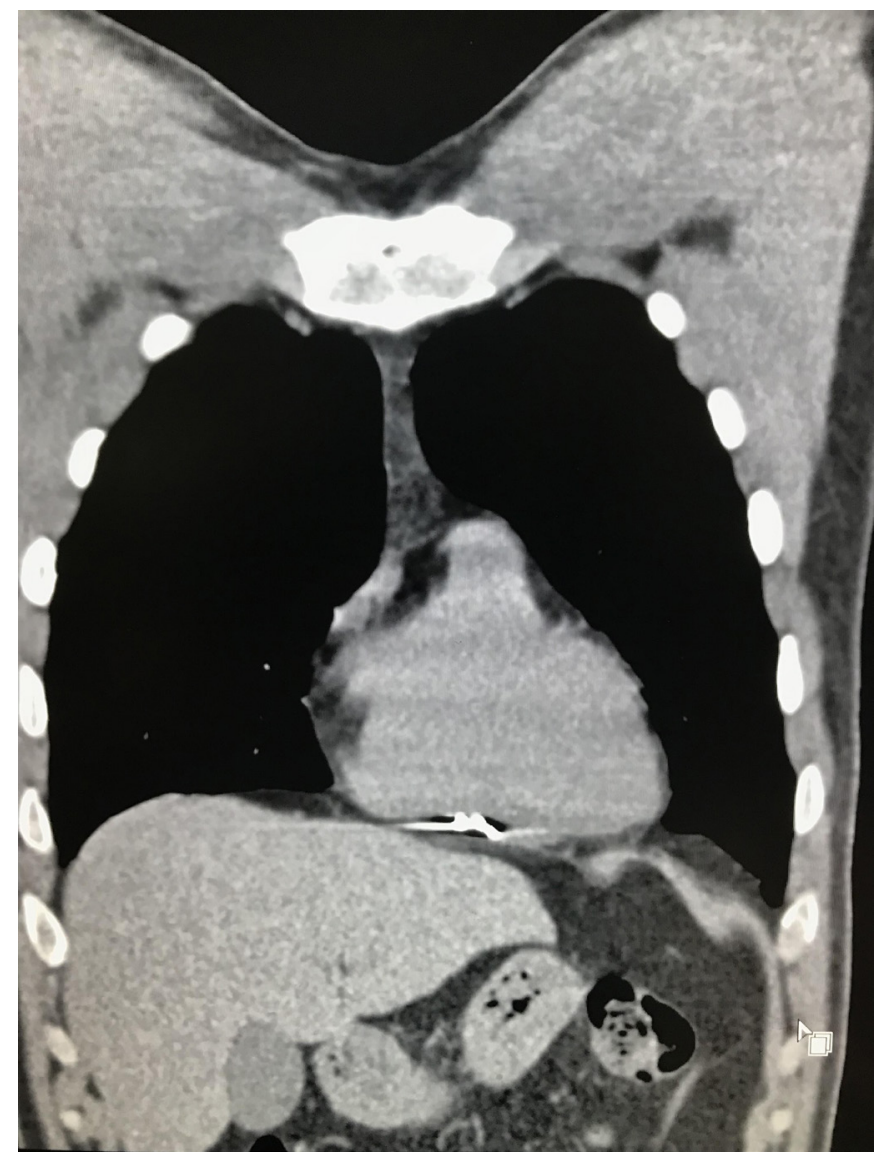

Figure 3.2. (coronal scan) Cardiac computed tomography showed the $\mathrm{FB}$ in right ventricular chamber.

Identification and the exact location of cardiac foreign bodies are crucial to treatment. However, the exact position of a metallic FB remains challenging. CT scanning

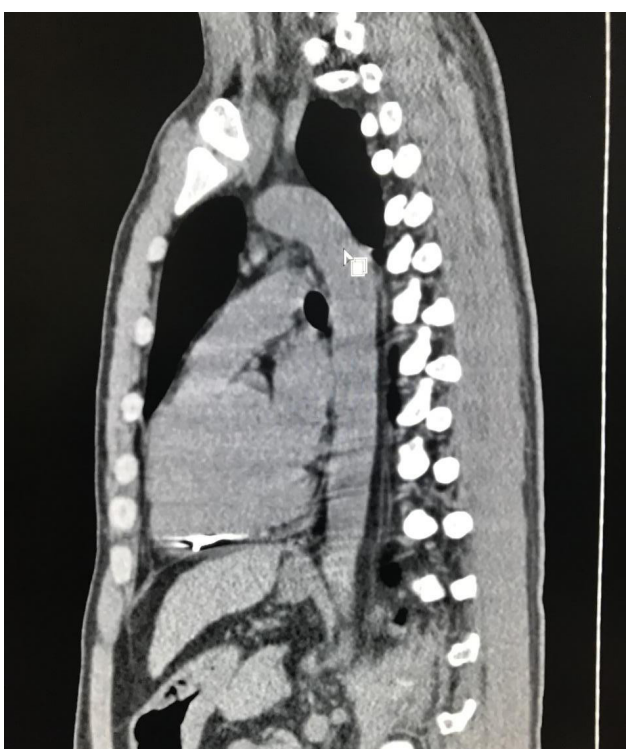

Figure 3.3. (sagittal reconstruction) Cardiac computed tomography showed the FB in right ventricular chamber.

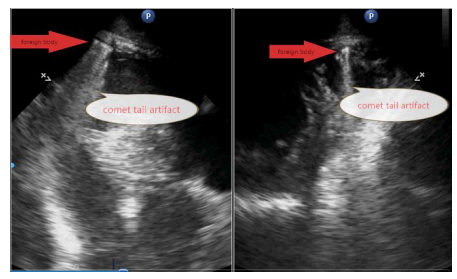

Figure 4. A nail in the apical right ventricular near the diaphragm in modified RV inflow view with special comet tail artifact.
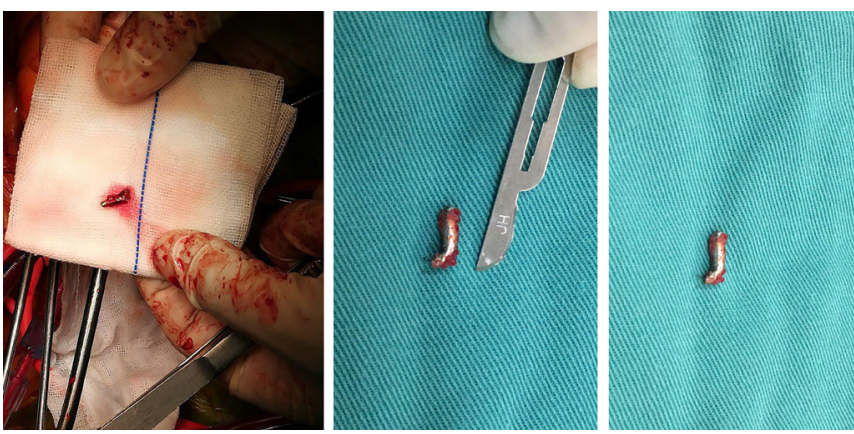

Figure 5. Removed nail covered by some clot.

was considered to be superior to 2D-TEE to demonstrate a metallic cardiac object-oriented in shape and direction. However, most of the FBs were located using TTE or through comprehensive TEE examination [Pan 2013; Qian 2015; Supomo 2018]. In this case, Bi-plane TEE was more accurate for its position, relationship, mobility, and size of the nail in the heart, which is characterized by reverberation artifact/comet-tail artifact. Besides, three-dimensional (3D)-TEE was suggested to assess an intracardiac metallic foreign body in the right heart without imaging artifacts 
from reverberations [Herbert 2018; Hsiung 2010]. In our case, however, 3D-TEE did not help localize the nail in the apex of the RV.

It was reported that bullet or missile fragments may enter the vascular system after a penetrating gunshot trauma and resulted in near-complete occlusion of the pulmonary artery, proximal dilation of pulmonary artery vasculature [Dell'Amore 2016; Unal 2017]. Gulel et al reported a lost guidewire entered the right atrium through the superior vena cava, passed into the right ventricle through the lateral tricuspid valve annulus, and then looped in the right ventricle; multiple mobile thrombi attached to a guidewire in the right atrium and right ventricle [Gulel 2013]. In our patient, although there was some clot on the surface of the nail, the patient was fortunate to avoid FB embolism to the pulmonary artery as it had lodged in the apex of the RV. Therefore, we thought identification of the FB's location and prompt removal of a metallic foreign body should be considered after an adequate assessment of the individual's situation.

\section{CONCLUSION}

We observed an accidental cardiac embolism of metallic FB (nail) that migrated from the jugular vein to the heart. Structural nail embolization to the heart is a rare entity that is reported here for the first time. The diagnosis of cardiac embolism should be considered when there is no evidence of an exit wound. Determination of the exact location of the FB in the heart was important to ensure complete and successful removal of the object. Bi-plane TEE offered great value for the localization of an intracardiac metallic FB with comet rail artifact.

\section{REFERENCES}

Actis Dato GM, Arslanian A, Di Marzio P, et al. 2003. Posttraumatic and iatrogenic foreign bodies in the heart:report of fourteen cases and review of the literature. J Thorac Cardiovasc Surg. 126(2):408-14.
Cianciulli TF, Mc Loughlin DE, Morita LA, et al. 2017. Bone cement cardiac and pulmonary embolism. Echocardiography. 34(8):1239-1241.

Dell'Amore A, Ammari C, Campisi A. et al. 2016. Peripheral venous catheter fracture with embolism into the pulmonary artery.J Thorac Dis. 8(12):E1581-E1584.

Di Paolo M, Guidi B, Vergaro G, et al. 2015. Self-Inserted Needles in the Heart.Am J Cardiol. 116(8):1315-7.

Gulel O, Soylu K, Yuksel S, et al. 2013. A forgotten guidewire causing intracardiac multiple thrombi with paradoxical and pulmonary embolism. Can J Cardiol. 29(6):751.e15-6.

Herbert JT, Kertai MD. 2018. Transesophageal Echocardiography Use in Diagnosis and Management of Embolized Intravascular Foreign Bodies. Semin Cardiothorac Vasc Anesth. 22(1):100-103.

Hsiung MC, Chang YC, Wei J. 2010. Embolization of the stent to the right heart after a motor vehicle accident. Echocardiography. 27(5):587-9.

Irdem A, Baspinar O, Gokaslan G. et al. 2015. Interesting case of sewing needle inside the heart: report of a case. Surg Today. 2015;45(4):503-5.

Liang H, Xu Z, Feng Q, et al. 2014. Recurrent cerebral embolism secondary to esophageal and atrial foreign body complicated by infective endocarditis. J Thorac Cardiovasc Surg. 148(5):e213-4.

Pan GZ, Bastidas JG, Hasaniya NW, Floridia R Jr. 2013. Bullet embolization from an aorto-caval fistula to the heart. Interact Cardiovasc Thorac Surg. 16(5):710-1.

Qian H, Song H, Li Y, Jiang C. 2015. Removal of metallic foreign body in heart by minimally invasive procedure under the guidance of transesophageal echocardiography and transthoracic echocardiogram. J Thorac Dis. 7(11):E560-3.

Supomo, Darmawan H. 2018. An Unusual Foreign Body in the Heart: A Case Report. Ann Thorac Cardiovasc Surg. 24(4):205-207.

Unal E, Balci S, Atceken Z, et al. 2017. Nonthrombotic Pulmonary Artery Embolism: Imaging Findings and Review of the Literature. AJR Am J Roentgenol. 208(3): 505-516. 